\title{
Electronic energy-density effects in ion tracks of metals
}

\author{
F. Staufenbiel, G. Schiwietz*, K. Czerski, M. Roth and
} P. L. Grande\#

Hahn-Meitner-Institut, Bereich Festkörperphysik,Glieniker Str. 100, D-14109 Berlin, Germany

\# Instituto de Física da Universidade Federal do Rio Grande do Sul, 91500 Porto Alegre, Brazil

*Corresponding author. Tel.: +49 308062 2448; fax: +49 30 8062 2293; e-mail: schiwietz@hmi.de

\begin{abstract}
High resolution Auger-electron spectroscopy has been applied to the interaction of swift heavy ions with atomically clean metallic solids. Spectra have been taken for fast projectile electrons and for charge-state equilibrated ions at normal incidence on micro crystalline beryllium samples, $\mathrm{Al}(100)$ single crystals and several metallic glasses $\left(\mathrm{Al}_{87} \mathrm{La}_{7} \mathrm{Ni}_{5} \mathrm{Zr}_{1}, \mathrm{Ni}_{78} \mathrm{~B}_{14} \mathrm{Si}_{8}, \mathrm{Co}_{66} \mathrm{Si}_{16} \mathrm{~B}_{12} \mathrm{Fe}_{4} \mathrm{Mo}_{2}\right)$. From the energy shift and from the Auger-line width we have extracted ion-track potentials and also electron temperatures inside ion tracks. A first determination of the angular distribution of multiple-ionization lines is presented as well.
\end{abstract}

PACS:79.20.Rf,31.70.Hq,79.20.Fv,72.20.Jv,32.80.Hd,72.15.Lh

keywords: electron temperature, ion-track potential,

multiple ionization, neutralization, recombination,

Auger decay, electron-spectra 


\section{Introduction}

Most of the qualitative results on electronic short time energy-density effects in ion-tracks are deduced from high resolution Auger-electron spectra. In this work, we extract electron temperatures from the line-shapes by comparing ion with electron induced spectra. Electron induced Auger-electron spectra correspond virtually to a cold electron gas around the projectile path.

In contrast to electrons, swift heavy ions can transfer energies of several hundred $\mathrm{eV}$ per target atom, deposited into the electron system of the target [1]. After some electron-electron collisions a local thermodynamic equilibrium may be reached. Very high electron temperatures can be achieved and electron cooling may be observed during the Auger-decay times of 1 to 10 fs [2]. The concept of a local thermodynamic heat-diffusion process for an electron temperature $T_{e}(t, d)$ as a function of the time $t$ after the ion passage and distance $d$ from the track center is based on two important requirements. Both requirements depend on the time $\tau_{\text {equi }}$ it takes to establish a local thermodynamic equilibrium energy-distribution, typically reached after a few inelastic collisions [3]. Firstly, $\tau_{\text {equi }}$ should be shorter than $\tau_{\text {Auger }}$, the mean Auger decay time at which the temperature of the track is probed. Only in this case we are allowed to think in terms of an electron temperature. Secondly, $\tau_{\text {equi }}$ should be shorter than $\tau_{\text {transport }}$, the mean transport time it takes for the electrons to diffuse from the track center to the infra-track radius. Only in this case we are allowed to think in terms heat diffusion, otherwise ballistic electron-transport processes are dominant.

$\tau_{\text {Auger }}$ depends on the type of transition and is tabulated for some transitions in this paper. $\tau_{\text {transport }}$ depends on the Fermi velocity, on the elastic scattering cross section and on the infra-track radius. It is typically 1 fs for primary ions at a few $\mathrm{MeV} / \mathrm{u} . \tau_{\text {equi }}$ depends on the material and on the electron temperature. Its value corresponds to 1 fs for many metals ( $\mathrm{Co}, \mathrm{Ni}, \mathrm{Fe}, \mathrm{Ta}$ ) and electron temperatures at 30000 to $40000 \mathrm{~K}$ [4]. For some metals (Cu, Ag), however, $\tau_{\text {equi }}$ is about 4 fs at the same temperature [4-5]. For these metals electron temperatures beyond $60000 \mathrm{~K}$ are necessary for equilibrium heat-diffusion processes. At very low temperatures $\left(T_{e}<20000 \mathrm{~K}\right)$ in an insulator or semiconductor electron-electron collisions completely loose importance and electrons dissipate their energy via the electron-phonon coupling. This corresponds to equilibration times $\tau_{\text {equi }}$ of a few hundred fs [6].

The position of the Auger-line maximum indicates any ion-track potential which could arise. This potential depends on the electronic properties of the target material, specifically on the electron trapping and on electronic conductivity given by the density and mobility of electrons and holes in all bands. Thus, the strongest track potentials are observed in insulators, i.e. in the polymers mylar and polypropylene [7], where electron trapping is strong and the electronic valence-band conductivity is virtually zero. For metallic solids, which have opposite electronic attributes, significant track potentials are not expected. However, this has to be proven experimentally.

The present work is the first systematic investigation of metals $(\mathrm{Al}, \mathrm{Be}$, and 
metallic glasses) with respect to electron temperatures and ion-track potentials. In a pioneering investigation [8] of clean silicon and aluminium samples neither any track potential nor any temperature effect was recognized. Moreover, for the first time an angular distribution of multiple-vacancy Auger lines induced by fast heavy ions is presented here.

\section{Experimental setup}

This investigation was performed at the Ionenstrahllabor (ISL) of the HahnMeitner-Institut in Berlin. The swift heavy ions get their high energy by successive acceleration in an electron cyclotron-resonance (ECR) source, a radio frequency quadrupole (RFQ) accelerator and in a sector-field cyclotron. The electron gun at the main chamber provides electrons with energies between 1 and $5 \mathrm{keV}$. Thus, electrons and heavy-ion projectiles have nearly the same velocity of about $10 \%$ of the speed of light. The emitted Auger electrons are energy selected and detected using an electrostatic parallel-plate spectrometer with an energy precision better than $0.2 \mathrm{eV}$. The evaluation concentrates on the high-energy slopes of the Auger lines. These contain the information on the electron temperature and consist of up to 20 data points. As described previously [9], ultra high vacuum is necessary to ensure that the atomically clean sputtered targets stay clean during the measurements.

For the measurement of the angular distributions of Auger electrons the spectrometer was rotated around the target in order to change the detection angle. The ejected electrons are detected in backward direction with respect to the incident ion beam.

\section{Results and discussion}

The considered Auger transitions are the so called CVV-Auger transitions (Core/Valence-band/Valence-band), where an inner-shell vacancy is filled with a valence band electron and the gained energy is transferred to another valenceband electron. The measurement of an electron temperature is possible because the valence-band population depends on the electron temperature via the FermiDirac distribution [10]. Figs. 1 and 2 show decomposed beryllium and aluminium Auger-spectra for incident ions and electrons, respectively. The multiple Auger lines correspond to different degrees of inner-shell ionization. The electron induced spectra show a steep high energy slope, because fast incident electrons can not excite multiple target electrons in the vicinity of an inner-shell vacancy. Therefore, we assume that we have a target-electron temperature of zero for incident electrons. 


\begin{tabular}{|c|c|c|c|c|}
\hline target & transition & $\tau_{\text {Auger }}$ & energy / ion & $\mathrm{T}_{e}\left[10^{4} \mathrm{~K}\right]$ \\
\hline$\overline{\mathrm{Be}}$ & Be-K $\mathrm{K}^{1} \mathrm{VV}$ & $14 \mathrm{fs}$ & $200 \mathrm{MeV}^{40} \mathrm{Ar}^{8+}$ & $2.0^{*}$ \\
\hline $\mathrm{Be}$ & Be-K ${ }^{1} V V$ & $14 \mathrm{fs}$ & $200 \mathrm{MeV}^{40} \mathrm{Ar}^{16+}$ & 3.4 \\
\hline$\overline{\mathrm{Be}}$ & $\mathrm{Be}-\mathrm{K}^{1} \mathrm{VV}$ & $14 \mathrm{fs}$ & $592 \mathrm{MeV}^{197} \mathrm{Au}^{48+}$ & 7.3 \\
\hline $\mathrm{Be}$ & Be-K ${ }^{2} V V$ & $7 \mathrm{fs}$ & $592 \mathrm{MeV}^{197} \mathrm{Au}^{48+}$ & 9.6 \\
\hline $\mathrm{Al}_{87} \mathrm{La}_{7} \mathrm{Ni}_{5} \mathrm{Zr}_{1}$ & $\mathrm{Al}-\mathrm{L}^{1} \mathrm{VV}$ & $15 \mathrm{fs}$ & $592 \mathrm{MeV}^{197} \mathrm{Au}^{48+}$ & $\geq 1.7^{*}$ \\
\hline $\mathrm{Al}(100)$ & $\mathrm{Al}-\mathrm{L}^{1} \mathrm{VV}$ & $15 \mathrm{fs}$ & $592 \mathrm{MeV}^{197} \mathrm{Au}^{48+}$ & $\geq 1.4^{*}$ \\
\hline $\mathrm{Al}(100)$ & $\mathrm{Al}-\mathrm{L}^{2} \mathrm{VV}$ & $8 \mathrm{fs}$ & $592 \mathrm{MeV}^{197} \mathrm{Au}^{48+}$ & $2.5^{*}$ \\
\hline $\mathrm{Al}(100)$ & $\mathrm{Al}-\mathrm{L}^{3} \mathrm{VV}$ & $5 \mathrm{fs}$ & $592 \mathrm{MeV}^{197} \mathrm{Au}^{48+}$ & 5.0 \\
\hline $\mathrm{Co}_{66} \mathrm{Si}_{16} \mathrm{~B}_{12} \mathrm{Fe}_{4} \mathrm{Mo}_{2}$ & $\mathrm{Si}^{-\mathrm{L}^{1} \mathrm{VV}}$ & $15 \mathrm{fs}$ & $592 \mathrm{MeV}^{197} \mathrm{Au}^{48+}$ & $\geq 5.4$ \\
\hline $\mathrm{Si}$ & $\mathrm{Si}^{-\mathrm{L}^{1} \mathrm{VV}}$ & $15 \mathrm{fs}$ & $592 \mathrm{MeV}^{197} \mathrm{Au}^{48+}$ & $\geq 4.9$ \\
\hline $\mathrm{Si}$ & Si-L ${ }^{2} V V$ & $8 \mathrm{fs}$ & $592 \mathrm{MeV}^{197} \mathrm{Au}^{48+}$ & 6.2 \\
\hline $\mathrm{Si}$ & Si-L $\mathrm{L}^{3} \mathrm{VV}$ & $5 \mathrm{fs}$ & $592 \mathrm{MeV}^{197} \mathrm{Au}^{48+}$ & 7.9 \\
\hline $\mathrm{Ni}_{78} \mathrm{~B}_{14} \mathrm{Si}_{8}$ & $\mathrm{Ni}-\mathrm{M}^{1} \mathrm{VV}$ & $0.4 \mathrm{fs}$ & $592 \mathrm{MeV}^{197} \mathrm{Au}^{48+}$ & $\geq 4.5$ \\
\hline $\mathrm{C}$ & $\mathrm{C}-\mathrm{K}^{1} \mathrm{VV}$ & $11 \mathrm{fs}$ & $1.9 \mathrm{GeV}^{238} \mathrm{U}^{68+}$ & 7.0 \\
\hline $\mathrm{C}$ & $\mathrm{C}-\mathrm{K}^{2} \mathrm{VV}$ & $6 \mathrm{fs}$ & $1.9 \mathrm{GeV}^{238} \mathrm{U}^{68+}$ & 8.5 \\
\hline
\end{tabular}

Table 1: Table 1 shows the semiconductors and metals investigated so far with the selected Auger transition (energies between 60 and $270 \mathrm{eV}$ ), the estimated Auger-decay time $\tau_{\text {Auger }}$, the projectile energy and ion species as well as the estimated electron temperature for this system. Temperatures $T_{e}$ are uncertain by about $\pm 10 \%$. For cases marked with an asterisk (*), we expect that local thermodynamic equilibrium is not reached because of ballistic heat transport. Results for carbon and silicon have been extracted from data taken previously $[2,9,10]$

For a rough estimate with the assumptions of a free electron gas and a convoluted density-of-states (DOS) with a Gaussian shape for the temperature broadening we can use $T_{e}=\frac{2}{3} \frac{1}{\sqrt{2} k} \sqrt{\Delta E^{2}-\Delta E_{0}^{2}}$ to estimate the electron temperature $T_{e}$, where $\Delta E$ is the peak width at half-height for the ion induced Auger-line, $\Delta E_{0}$ the peak width at half-height for the electron induced Augerline and $k=8.617342 \cdot 10^{-5} \frac{\mathrm{eV}}{\mathrm{K}}$ the Boltzmann constant [2]. The electron temperatures determined in this way are summarized in Table 1 together with the Auger-decay time corresponding to each specific transition [11, 12].

For some target materials we can get information about the time dependent temperature variation. In all cases we found a decreasing electron temperature as a function of time. Therefore, the maximum temperature corresponds to very short times. The analysis of metallic alloys compared to crystalline metals yields information about the influence of the atomic structure on the electron temperature. Here we may compare the $\mathrm{Al}(100)$ single crystal with the $\mathrm{Al}_{87} \mathrm{La}_{7} \mathrm{Ni}_{5} \mathrm{Zr}_{1}$ glass as well as the $\mathrm{Co}_{66} \mathrm{Si}_{16} \mathrm{~B}_{12} \mathrm{Fe}_{4} \mathrm{Mo}_{2}$ glass, selecting the silicon Auger transition, with the electron temperature in a Si (111) single crystal. In the amorhpous lattices the electron temperature is a little higher because the specific electron heat conductivity is reduced. For the analysis of DOS effects in the measured electron temperature we have chosen micro crystalline beryllium 
and an $\mathrm{Al}(100)$ single crystal. In contrast to beryllium, with its very low DOS at the Fermi energy [13], aluminium is a free-electron gas like metal. The temperature of beryllium is roughly a factor of four higher than the one determined for aluminium for the same type of incident ions and at a similar Auger-decay time. This is a clear signature of the DOS affecting the electronic heat capacity $\mathrm{C}_{e}$ (the dominant parameter for small times) and correspondingly $\mathrm{T}_{e}$. To within an uncertainty of $\pm 0.4 \mathrm{eV}$ non of the investigated metals has shown an energy shift corresponding to an ion-track potential.

Auger spectra for beryllium have also been taken in backward directions at spectrometer angles ranging from $115^{\circ}$ to $160^{\circ}$ with respect to the incident ion beam. Fig. 3 shows the ratio of the integrated Auger-line intensities, with the $\mathrm{K}^{2} \mathrm{VV}$ yields divided by the $\mathrm{K}^{1} \mathrm{VV}$ yields. For increasing ejection angles the ratio decreases significantly. For light projectiles such as electrons, it is well known that the angular distributions for amorphous samples do closely resemble cos-functions [14]. Thus, the ratio of two neighboring lines should be almost constant. However, the integrated Auger-line ratios should be sensitive to the spatial hot-electron regime, because most other angular dependences are eliminated by calculating the ratio. The enhanced temperature for the $\mathrm{K}^{2} \mathrm{VV}$ decay (see Table 1) might specifically influence the corresponding angular distribution and lead to reduced intensities along the normal surface direction. A similar reduction is found in a preliminary evaluation of $\mathrm{Al}-\mathrm{L}^{n} \mathrm{VV}$ Auger intensities.

\section{Conclusions}

Fast heavy ion induced Auger spectra of metals have been taken with high resolution. The key point of this investigation is the determination of electron temperatures related to the energy transfer by projectile ions. Electron temperatures have been extracted from the shape of the Auger-line structures, resulting in values between $10000 \mathrm{~K}$ and $100000 \mathrm{~K}$ for multiple degrees of ionization corresponding to Auger-decay times around $10 \mathrm{fs}$. Furthermore, there is no significant sign of ion-track potentials for the metallic samples, as the corresponding Auger-energy shifts have not been found. Hence, conduction band electrons do rapidly neutralize the atomic charges inside the track and therefore, Coulomb explosion is impossible.

Furthermore, angular distributions of fast heavy-ion induced Auger electrons from beryllium and aluminium have been measured for the first time and significant ion-track effects have been found for the line-intensity ratios. In a highly excited electron system the mean free electron path-length may eventually be reduced. The geometry of the ion track leads then to a suppression of Augerelectron yields along the surface normal. In conclusions, significant influences of the high electronic energy density on the short-time electron dynamics of metals have been found and partially identified for the first time. 


\section{References}

[1] P.L.Grande, G.Schiwietz, Nucl. Instr. and Meth. B 195 (2002) 55-63

[2] G.Schiwietz, G.Xiao, P.L.Grande, E.Luderer, R.Pazirandeh,U.Stettner, Europhys. Lett. 47 (1999)

[3] Eduardo M. Bringa, Lawrence Livermore National Laboratory (private communication)

[4] M. Bauer, M. Aeschlimann, Journal of Electron Spectroscopy and Related Phenomena 124, 225-243 (2002)

[5] T. Hertel, E. Knoesel, M. Wolf, and G. Ertl, Phys. Rev. Lett. 76/3, 535-538 (1996)

[6] A. J. Sabbah and D. M. Riffe, Phys. Rev. B 66, 165217 (2002)

[7] G.Schiwietz, P.L.Grande, B.Skogvall, J.B.Biersack, R.Köhrbrück, K.Sommer, A.Schmoldt, P.Goppelt, I.Kádár, S.Ricz, U.Stettner, Phys. Rev. Letters Vol.69., Nr.4 (1992) 628-631

[8] W.Schmidt, P.Müller, V.Brückner, F.Löffler, G.Saemann-Ischenko, W.Schubert, Phys.Rev. A 24 (1981) 2420

[9] G.Schiwietz, K.Czerski, M.Roth, F.Staufenbiel, E.Luderer, P.L.Grande, Nucl. Instr. and Meth. B 193 (2002) 705-712

[10] G.Schiwietz, G.Xiao, P.L.Grande, E.Luderer, R.Pazirandeh,U.Stettner, Nucl. Instr. and Meth. B 146 (1998) 131-136 4282

[11] P.H.Citrin, G.K.Wertheim, Phys.Rev. B Vol.16, Nr.10 (1977) 4256-

[12] M.Krause, J.H.Oliver, J.Phys.Chem.Ref.Data 8, (1979) 329 384-390

[13] G.Pari, V.Kumar, A.Mookerjee, A.K.Bhattacharyya, J.Phys.: Condens.Matter 11 (1999) 4291-4302

[14] W.S.M.Werner, H.Tratnik, J.Brenner, H.Störi, Surface Science 495 (2001) 107-119 


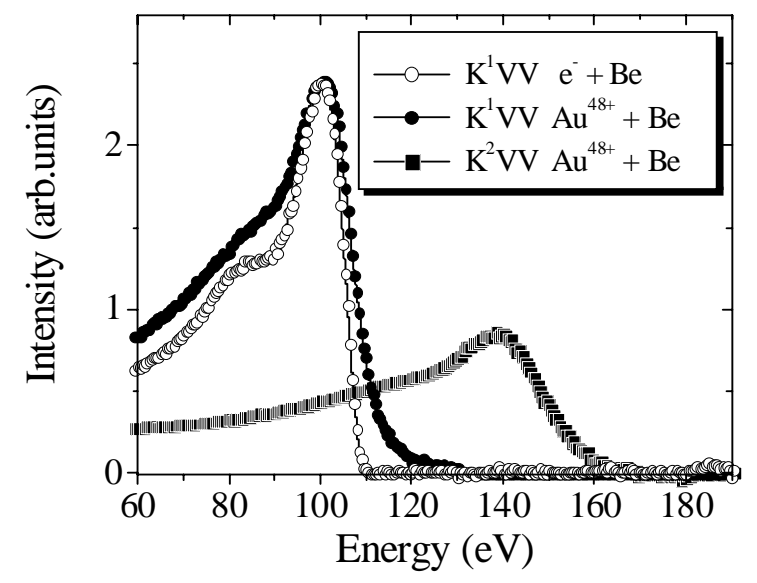

Figure 1: Beryllium Auger-spectra at $\theta_{e}=150^{\circ}$ after background subtraction. Auger structures are decomposed into different vacancy configurations $\left(K^{n} V V\right)$ for incident Au ions at $592 \mathrm{MeV}$ and electrons, respectively.

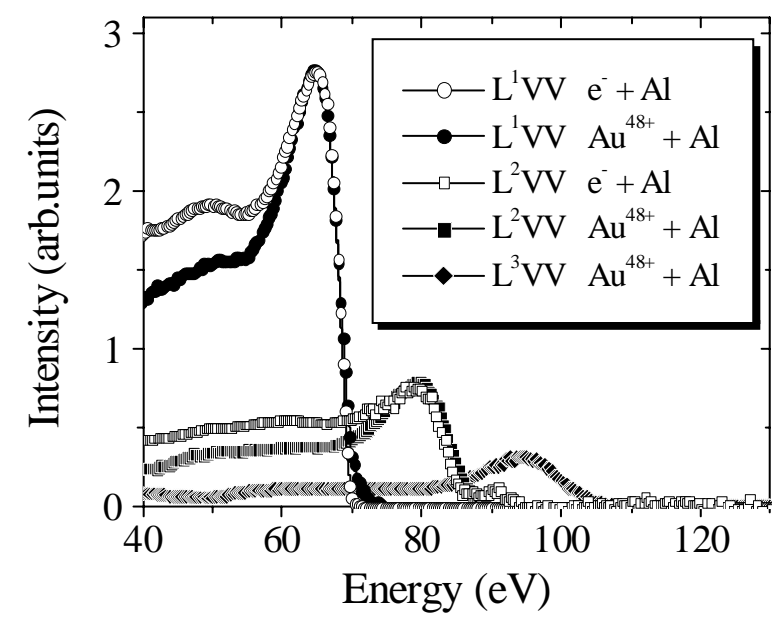

Figure 2: Aluminium Auger-spectra at $\theta_{e}=140^{\circ}$ after background subtraction. Auger structures are decomposed into different vacancy configurations $\left(L^{n} V V\right)$ for incident Au ions at 592 $\mathrm{MeV}$ and electrons, respectively. 


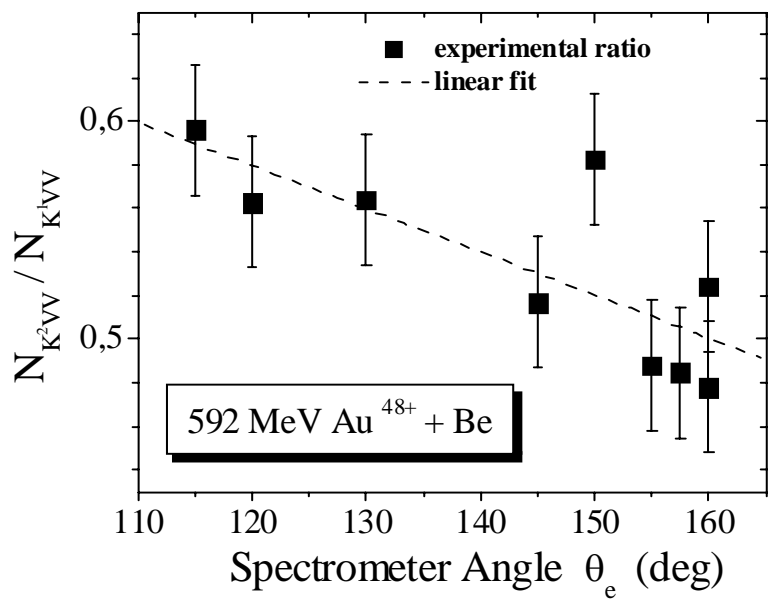

Figure 3: Ratio of integrated Auger-intensities for different vacancy configurations in micro crystalline beryllium. 